\title{
Topiramate in the treatment of partial and generalized epilepsy
}

\author{
Edward Faught \\ Department of Neurology, University \\ of Alabama School of Medicine, \\ Birmingham, Alabama, USA
}

\begin{abstract}
Topiramate (TPM) is a widely-used drug for the treatment of epilepsy. It is useful for several types of partial-onset and generalized-onset seizures, and is therefore considered a broad-spectrum agent. It is also effective as a prophylactic against migraine headaches. TPM was first approved for prescription use in 1996. In various countries it is now approved for adjunctive and monotherapy of partial-onset seizures and for therapy of generalized tonic-clonic seizures of nonfocal origin, for children and adults. For initial monotherapy of new-onset seizures, a target dose of $100 \mathrm{mg} /$ day for adults is recommended. Adjunctive use with enzyme-inducing drugs and use for refractory seizures requires higher dosages, though the optimum dose for most patients does not exceed $400 \mathrm{mg}$ /day. Excretion is primarily renal and TPM is not a significant hepatic enzyme inducer. Although it is usually safe and well-tolerated, adverse effects limit use in about $25 \%$ of patients. The most salient of these is cognitive dysfunction, especially problems with expressive speech and verbal memory. Weight loss, renal stones, paresthesias and other central nervous system side effects may occur. Tolerability is improved by low initial doses and slow titration to effect.
\end{abstract}

Keywords: epilepsy, seizures, antiepileptic drugs, topiramate

\section{Introduction}

For almost twenty years between the mid-1970s, when carbamazepine and valproic acid were introduced, and 1993, when felbamate was approved, no new antiepilepsy drugs were developed for clinical use. In the past 13 years, however, ten new drugs have become available. Topiramate is one of the more important of these second-generation drugs. In spite of this greatly-expanded range of therapeutic options, about $30 \%$ of patients remain refractory to medical therapy (Kwan and Brodie 2000), though 5\% are good candidates for surgery. Optimal use of topiramate and other newer drugs offers the possibility of better control for refractory patients, and often provides advantages in terms of ease of use and reduction of side effects. This review emphasizes the results of randomized controlled clinical trials of TPM and practical considerations for maximizing efficacy and minimizing adverse effects.

\section{Pharmacology}

TPM is a fructose derivative (2,3:4,5-bis- $O$-(1-methylethylidene)-D-fructopyranose sulfamate) (Figure 1). It was synthesized as part of a search for compounds with hypoglycemic activity (Maryanoff et al 1987). Although it has no significant direct effects on blood sugar, it was found to be effective in several animal seizure models (Shank et al 2000). These include models predictive of efficacy against several human seizure types: tonic-clonic seizures - the rodent maximal electroshock model (Shank et al 1994), complex partial seizures - amygdalar kindling (Wauquier and Zhou 1996), and absence seizures - genetic absence epilepsy rats (Rigoulot et al 2003). 


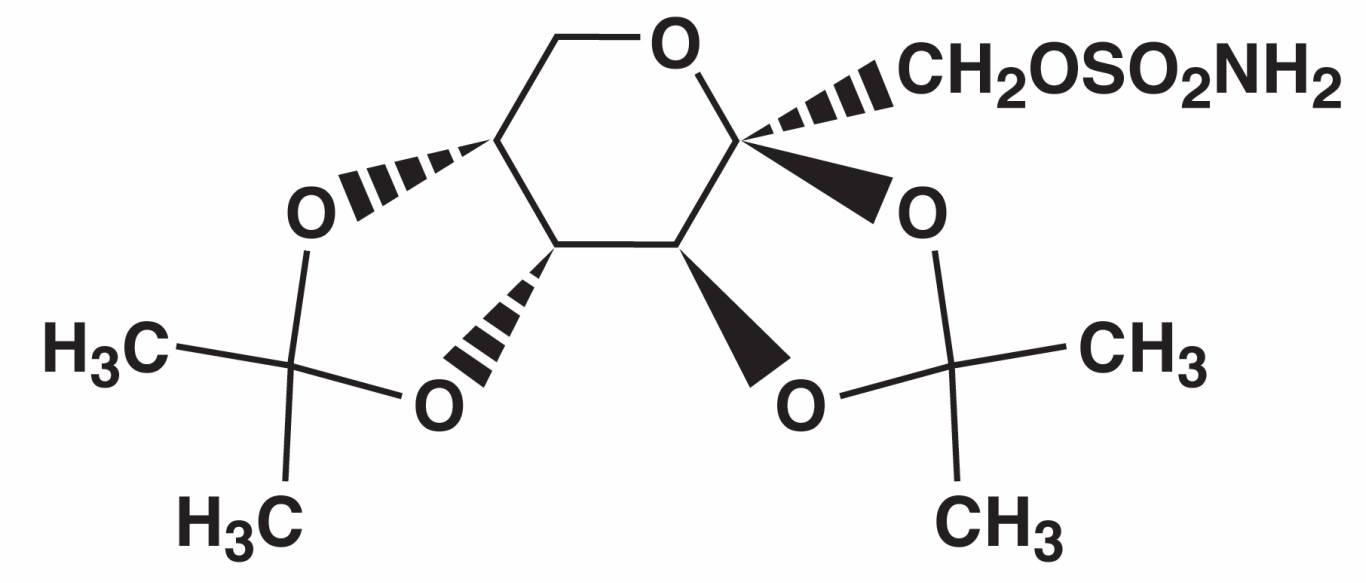

Figure I Structure of topiramate.

\section{Mechanisms of action}

The broad spectrum of activity against various seizure types may reflect multiple mechanisms of action. Mechanisms which have been identified are:

1. voltage-gated sodium channel blockade (Coulter et al 1993; McLean et al 2000)

2. kainate-type glutamate receptor inhibition (Gryder and Rogawski 2003)

3. reduction of L-type voltage-sensitive calcium currents (Zhang et al 2000)

4. increased frequency of GABA-mediated chloride channel opening (White et al 1997; White et al 2000)

5. carbonic anhydrase inhibition (Dodgson et al 2000)

6. increased potassium conductance (Herrero et al 2002)

The first three actions would serve to reduce neuronal excitation, the last three would enhance inhibition. It is not known which of these proposed mechanisms is most important for the antiseizure effects. Neuroprotective effects against damage from status epilepticus (Niebauer and Gruenthal 1999) and from hypoxia (Koh and Jensen 2001) have also been described.

\section{Pharmacokinetics}

Linear pharmacokinetics make TPM dosing straightforward. It is rapidly and relatively completely ( $80 \%$ bioavailability) absorbed after oral dosing (Doose et al 1996). The maximum concentration (Cmax) is achieved about 2 hours after an oral dose (range 1.4-4.3 hours) (Doose et al 1996); consequently this is the time of day at which dose-related adverse effects are most likely to occur. About $60 \%$ of a dose is excreted unchanged by the kidneys; the rest undergoes hepatic metabolism to several inactive metabolites (Wu et al 1994; Johannessen 1997). With normal liver and kidney function in monotherapy the elimination half-life $\left(T^{1 / 2}\right)$ is about 20-30 hours after single or multiple doses; thus four or five days are required to reach a steady-state serum level with constant daily dosing (Doose et al 1996; Perucca 1997). Renal insufficiency diminishes clearance and prolongs the elimination half-life: patients with creatinine clearances of $<30 \mathrm{~mL} / \mathrm{min}$ experienced about a twofold increase in serum TPM levels (Gisclon et al 1993). Elderly patients also require reduced doses because of their expected slightly reduced renal function. Clearance in children is faster by 
approximately 50\% (Rosenfeld et al 1999) and is even faster in infants (Glauser 1999); increased doses on a milligramper-kilogram basis may therefore be required. Measurement of serum levels may be helpful in establishing an appropriate daily dose in these special populations.

\section{Drug interactions}

\section{Effects of topiramate on other drugs}

TPM has little effect upon the pharmacokinetics of other drugs because it is not a strong enzyme inducer nor inhibitor (Sachdeo et al 1996; Doose, Brodie et al 2003; Bialer et al 2004). However, in three patients with baseline serum phenytoin levels of $15 \mu \mathrm{g} / \mathrm{ml}$ or more, phenytoin levels rose $30 \%-50 \%$ when high doses of TPM (400-800 mg/day) were added (Sachdeo, Sachdeo et al 2002). This effect, which may be mediated by an inhibitory influence of TPM on cytochrome P450 2C19, could cause symptoms of phenytoin toxicity. Theoretically, the same effect could raise diazepam levels but this has not been reported. Proteinbinding interactions, such as may occur with phenytoin and valproate, are not a problem because TPM is only minimally protein bound. Rare cases of hyperammonemic encephalopathy have been observed with combination topiramate-valproate therapy. This phenomenon does not seem to be related to a pharmacokinetic interaction since TPM produces little net change in valproate serum levels, but it could be related to a shift in valproate metabolic pathways toward the 4-ene metabolite in the presence of TPM (Hamer et al 2000).

TPM may interfere with the contraceptive effect of birth control pills by inducing the metabolism of estrogen. In one study, doses of $200 \mathrm{mg}$ /day or more reduced ethinyl estradiol levels derived from oral contraceptives by $30 \%$ (Rosenfeld et al 1997). However, progesterone levels are not affected (Doose, Wang et al 2003), and the risk of unwanted pregnancy may therefore be lower than with drugs such as phenytoin and carbamazepine which induce the metabolism of both hormones. Women should be cautioned about the possibility of birth control failure. Use of a second or an alternate method or of a higher dose of estrogen may be considered.

TPM slightly decreases the serum levels of amitryptiline, lithium, and risperidone (Bialer et al 2004). Effects on other psychoactive agents are unknown. TPM is often used as a prophylactic against migraine; it is helpful to know that it has no pharmacokinetics effects upon the antimigraine agents dihydroergotamine, propranolol, and sumatriptan (Bialer et al 2004).

\section{Effects of other drugs upon topiramate}

In contrast to the minor effects of TPM on other drugs, enzyme-inducing drugs have major influences on the disposition of TPM. Phenytoin and carbamazepine each double the clearance of TPM and therefore reduce TPM serum levels by up to 50\% (Sachdeo, Sachdeo et al 2002; Neurologics 2006). Barbiturates likely have a similar effect but have not been studied. Conversely, when one of these drugs is withdrawn from a combination regimen, TPM levels will rise and this could result in dose-related adverse effects. However, this is often offset by the improved tolerability resulting from removal of the concomitant drug.

\section{Efficacy: partial-onset seizures Refractory partial-onset seizures:} adjunctive therapy

As with all antiepilepsy drugs developed within the past twenty years, TPM was first tested in populations of patients with refractory partial-onset seizures, as an adjunctive agent. These patients are the most numerous in epilepsy treatment centers.

The first trials in the United States enrolled adults taking one or two concomitant antiepilepsy drugs. Early results established excellent efficacy at all daily dosages of $200 \mathrm{mg}$ or more (Faught et al 1996; Privitera et al 1996). Dosages of $600-1000 \mathrm{mg} /$ day conferred no additional reductions in seizure frequency but caused more adverse effects. The optimum dose of $400 \mathrm{mg} /$ day produced a "responder rate" - the percentage of patients experiencing at least a $50 \%$ reduction in seizure number from their baseline - of $41 \%$ (Faught et al 1996). Additional studies with comparable populations carried out in Europe, Korea, and Canada confirmed these results and established that lower dosages of $200-400 \mathrm{mg} /$ day are effective as adjunctive therapy (Ben-Menachem et al 1996; Sharief et al 1996; Tassinari et al 1996; Korean Topiramate Study Group 1999; Guberman et al 2002). A clinical trial in children aged 1-16 years produced a similar result, with a median percent reduction from baseline in seizure frequency of $33 \%$ compared to $11 \%$ with adjunctive placebo (Elterman et al 1999).

Open-label studies do not suggest excessive development of tolerance to the antiepilepsy effects of TPM with longterm treatment, with retention rates on drug of $30 \%-50 \%$ for 3-4 years, most dropouts being due to adverse effects (Abou-Khalil 2000; Lhatoo et al 2000; Bootsma et al 2004). However, it is probable that tolerance develops in some patients with refractory epilepsy treated with any antiepilepsy drug (Loescher and Schmidt 2006). 


\section{Refractory partial-onset seizures: monotherapy}

Adults with four or more seizures per month despite taking one or two drugs were gradually converted to monotherapy with a low dose $-100 \mathrm{mg} /$ day - or a very high dose $-1000 \mathrm{mg} /$ day - of TPM as the baseline drugs were simultaneously withdrawn (Sachdeo et al 1997). Patient safety was addressed by stopping rules such as a doubling of baseline seizure frequency or the new occurrence of a tonic-clonic seizure. This trial was designed to demonstrate TPM efficacy as monotherapy, not to establish a recommended monotherapy dose or to quantify improvement in comparison to previous treatment. The study achieved this goal, as significantly more of the high-dose subjects were converted successfully without invocation of a stopping rule $(\mathrm{p}<0.01)$.

\section{Partial and generalized new-onset seizures: monotherapy}

Two clinical trials of TPM monotherapy employed a similar design, with children and adults with presumed partial-onset seizures being randomized to a lower or to a higher dose. In the first trial (Gilliam et al 2003), 252 patients aged 3 years or more took a low dose $(50 \mathrm{mg} /$ day for those weighing $50 \mathrm{~kg}$ or more, $25 \mathrm{mg}$ /day for those weighing $<50 \mathrm{~kg}$ ) or a high dose $(500 \mathrm{mg}$ /day for weight of $50 \mathrm{~kg}$ or more, $200 \mathrm{mg} /$ day for weight $<50 \mathrm{~kg}$ ). Doses were adjusted only for weight, not for age. Fifty-six percent of subjects were on no antiepilepsy drug; the others were already taking one drug (usually phenytoin) which was withdrawn over the first six weeks. Results were inconclusive for time to the first seizure, but favored the high dose $(p<0.01)$ when time to the second seizure was added as a covariate. The second trial (Arroyo et al 2005) used an identical design except that patients 6 years and older were enrolled and the low-dose target was $50 \mathrm{mg}$ /day, the high-dose target $400 \mathrm{mg} /$ day. Time to the first seizure favored the high dose, with seizure-free rates of $59 \%$ and $76 \%$ for the low and high doses respectively $(\mathrm{p}=0.001)$.

It is often difficult to determine during the initial evaluation of a patient with new-onset seizures whether they are of partial or generalized origin. This is especially true if the event was a convulsion - a generalized tonic-clonic seizure, which could have originated locally or generally. For this reason, most new-onset therapeutic trials include a mixture of seizure types. This is obviously undesirable but does reflect the reallife clinical situation. Recognizing this difficulty, investigators designed a study allowing for entry of all new-onset seizure patients, but with a provision for varying the control drug by the physician's choice (Privitera et al 2003). In this study, monotherapy doses of TPM were employed in a three-way randomized, blinded comparison to valproate and to carbamazepine. A novel feature of this trial was the permission for the treating physician to select either valproate or carbamazepine as the control agent. It was anticipated that if generalizedonset seizures were suspected, valproate would be chosen, and if partial-onset seizures were suspected, carbamazepine would be chosen. This was usually, but not always, the case, in part reflecting the difficulty physicians have in making this distinction early in the course of the disorder. After this choice, patients were randomized to receive TPM $100 \mathrm{mg} /$ day, TPM $200 \mathrm{mg} / \mathrm{day}$, carbamazepine $600 \mathrm{mg} /$ day, or valproate 1000 $\mathrm{mg} /$ day. All treatments proved equally effective, thus TPM $100 \mathrm{mg} /$ day was recommended by the authors as the initial target dose for patients with new-onset seizures. A word of caution is necessary in interpreting new-onset seizure drug comparisons: many patients would achieve good control regardless of the drug chosen, some would be refractory from the outset. The presence of these two categories of patients in significant numbers would tend to reduce the sensitivity of the study to detect differences in efficacy. Indeed, nearly all such comparisons in recent years have shown efficacy to be equivalent between many pairs of drugs; larger sample sizes may be needed to detect any true differences.

In order to address the problem of sample size in newonset epilepsy, the recent SANAD (Standard And New Antiepileptic Drugs) study in the United Kingdom employed a very large number of investigators to enroll 1721 patients with partial epilepsy and 716 with generalized or unclassifiable epilepsy (Marson et al 2007a, 2007b). This study was randomized but unblinded, and allowed considerable dosing flexibility. Lamotrigine was found to be the best drug for partial epilepsy based on "time to treatment failure" but its superiority over carbamazepine, oxcarbazepine, and topiramate was entirely due to superior tolerability; there were no significant differences in efficacy of seizure control among these drugs (Marson et al 2007a). Similarly, in the generalized epilepsy study, valproate was deemed superior to lamotrigine based on efficacy, but its superiority to topiramate was based on tolerability; the efficacy of valproate and topiramate was equivalent (Marson et al 2007b).

\section{Generalized-onset seizures Generalized tonic-clonic seizures of nonfocal origin}

TPM is effective against generalized tonic-clonic seizures "grand mal" - whether of partial origin ("secondarily generalized") (Faught et al 1996, Privitera et al 1996) or of generalized origin ("nonfocal origin"). The latter condition 
was investigated in a double-blind placebo controlled study of 80 patients aged 3 to 59 years (Biton et al 1999). The median baseline seizure frequency was 4.5 per month for the placebo group and 5.0 per month for the TPM group: these patients had quite severe epilepsy. The median TPM dose achieved during the stable dose period was $5 \mathrm{mg} / \mathrm{kg} /$ day, a moderate dose. The median percent seizure reduction was $57 \%$ for adjunctive TPM and $9 \%$ for adjunctive placebo $(p=0.019)$. The responder rate $(50 \%$ or better reduction in seizure frequency) was $56 \%$ for TPM and $20 \%$ for placebo $(\mathrm{p}=0.001)$.

\section{Other primary generalized epilepsies}

Primary generalized epilepsies are syndromes producing generalized-onset seizures but are otherwise associated with little or no neurological dysfunction. They are probably of genetic origin and typically appear at ages characteristic for each syndrome. Few data concerning the effect of TPM in these syndromes are available. Small open-label series suggest efficacy in childhood absence epilepsy (Cross 2002) and juvenile myoclonic epilepsy (Biton and Bourgeois, 2005, Sousa Pda et al 2005). Since juvenile myoclonic epilepsy is a common cause of convulsions in teenagers and young adults, it is probable that many of the patients in the study of generalized tonic-clonic seizures of nonfocal origin (Biton et al 1999) had this syndrome.

\section{Secondary generalized epilepsies}

This category of the International Classification of Epilepsies and Epilepsy Syndromes includes conditions characterized by moderate to severe underlying brain pathology with epilepsy as a prominent symptom. Many of these patients have intellectual disabilities or other neurological deficits and their seizures are often refractory to treatment. The most common of these diseases is the Lennox - Gastaut syndrome.

\section{Lennox - Gastaut syndrome: adjunctive treatment}

A triad of atypical absence seizures, intellectual disabilities, and a "slow spike-and-wave" EEG pattern typifies this syndrome, which usually begins in early childhood. Most patients have other seizure types, especially "drop attacks" - a term subsuming atonic, axial tonic, and generalized tonic-clonic seizures. Valproate is a drug of choice but frequently fails to control seizures adequately. Ninety-eight patients aged 2-42 (mean age 11) were randomized to adjunctive placebo or adjunctive TPM; a mean TPM dosage of $5.8 \mathrm{mg} / \mathrm{kg} /$ day was achieved (Sachdeo et al 1999). Baseline therapy usually included valproate. Drop attacks were reduced 15\% in the TPM group; they increased 5\% in the placebo group ( $p=0.041$ ). Fifty-two percent of TPM patients vs $38 \%$ of placebo patients experienced an improvement in seizure frequency as judged by parents $(\mathrm{p}=0.040)$.

\section{Other secondary generalized epilepsies}

Except for the Lennox - Gastaut syndrome, none of the secondary generalized epilepsies have been the subject of controlled clinical trials. Most are infrequent or rare.

\section{Infantile spasms (West syndrome)}

Infantile spasms (West syndrome) are ominous events; they often occur as precursors to the Lennox - Gastaut syndrome and are associated with a high rate of intellectual disability in later life. The diagnosis is most commonly made during the second half of the first year of life. Several small open-label studies have reported improvements in some patients with infantile spasms and in the associated hypsarrhythmic EEG abnormality with TPM treatment (Glauser et al 1998; Thijs et al 2001; Grosso et al 2005; Valencia et al 2005; Hosain et al 2006).

\section{Dravet's syndrome}

Dravet's syndrome is also known as severe myoclonic epilepsy of infancy. Four open-label reports, collectively including 57 patients, reported good improvement in 31 cases (Nieto-Barrera et al 2000; Coppola et al 2002; Mikaeloff et al 2003; Grosso et al 2005).

\section{Progressive myoclonic epilepsies}

Progressive myoclonic epilepsies are syndromes characterized by myoclonic and generalized tonic-clonic seizures and progressive cerebral dysfunction. Their etiologies are heterogeneous. Valproate is usually prescribed for the seizures; TPM may be a useful adjunct (Aykutlu et al 2005).

\section{Status epilepticus}

TPM has been used effectively in some cases of status epilepticus refractory to other agents (Towne et al 2003). Since there is no parenteral form, tablets must be crushed and administered by nasogastric tubing.

\section{Adverse effects}

Serious adverse effects of TPM are rare, but effective use requires familiarity with several common mild to moderate effects. Adverse effects resulted in discontinuation of TPM in $11 \%-28 \%$ of patients during brief adjunctive therapy clinical trials (Reife et al 2000); this is within the higher range of discontinuations of most other new antiepilepsy drugs tested 
under similar circumstances (Faught and Limdi 2002). The most common reason for discontinuation is the occurrence of cognitive problems (Tatum et al 2001; Bootsma et al 2004). However, the persistence rate - the percentage of patients still taking TPM 36 three years after initiation, is $30 \%$, in the higher range of retention for new antiepileptic drugs (Lhatoo et al 2000). These data suggest that if patients tolerate TPM treatment early in treatment, they have a good chance of continuing to have satisfactory results for a long time.

\section{Cognitive effects}

Expressive language difficulty, especially word-finding difficulty and mild dysnomia, is common, and may be more prevalent in patients with pre-existing dominant hemisphere dysfunction (Mula et al 2003). This effect is also seen in normal volunteers given topiramate (Meador et al 2005). The degree of language dysfunction varies from a minor annoyance to a significant problem, and may be unnoticed by the patient. This effect is rather specific for TPM and is rarely seen with other antiepilepsy drugs apart from the setting of a generalized cognitive impairment; with TPM it may be the only side effect. Verbal memory impairments can be demonstrated in some patients (Aldenkamp 2000; Aldenkamp et al 2000; Meador et al 2003), especially to immediate working memory (ie, digit span) (Lee et al 2006).

More global cognitive difficulties may occur. In the early, high-dose, rapid titration adjunctive therapy clinical trials "thinking abnormal" (a rather nonspecific World Health Organization coding term) was reported in $13 \%-33 \%$ and "concentration impaired" in 15\%-25\%; often these were in the same patients (Reife et al 2000). These effects are clearly dose-related (Shorvon 1996). In a study of lowerdose adjunctive TPM, $200 \mathrm{mg} /$ day, using a slow titration rate of $25-50 \mathrm{mg}$ /day increases weekly, only $5 \%$ of patients experienced "concentration/attention difficulty" (Guberman et al 2002).

Monotherapy is better tolerated, with reductions in the probability of individual cognitive side effects to below $10 \%$ in most series (Sachdeo et al 1996; Gilliam et al 2003; Arroyo et al 2005). Cognitive adverse effects of TPM are not inevitable. Even at higher doses, many patients are unaffected; group differences are strongly influenced by a subset of patients significantly impaired (Meador et al 2003).

On the other hand, there is one report that low-dose monotherapy may cause subjective and objective cognitive problems: dose-related decrements in verbal fluency and working memory (as measured by digit span) displayed a clear dose-response relationship to doses of 75, 100, and
$200 \mathrm{mg} /$ day in an open-label monotherapy series of 36 epilepsy patients (Lee et al 2006). Moreover, these effects were present even after one year of treatment, at which time $44 \%$ of the patients had cognitive complaints, though most were mild. In this study the $50 \mathrm{mg} /$ day dose did not affect cognitive function, and many patients chose to continue the higher doses anyway because of good seizure control.

The mechanism of the cognitive side effects is unclear; they are often observed independently of changes in level of alertness or mood. Functional magnetic resonance imaging (fMRI) revealed decreased activation of prefrontal cortex in response to a verbal task in normal volunteers who took TPM (Jansen et al 2006), but the biochemical mechanism is unknown.

Effects on cognition may be subtle and may not be volunteered by the patient: it is necessary to search for them by directed questioning, and it is often helpful to interview the family.

\section{Weight loss and anorexia}

TPM therapy causes an average decrease in body weight of $4.6 \%$. This is apparently the result of anorexia and weight tends to stabilize by 18 months of treatment (Rosenfeld and Slater 2002; Ben-Menachem et al 2003). Obese patients may lose more. This is often a benefit but can limit therapy in patients who cannot voluntarily increase their caloric intake and in children.

\section{Somnolence and fatigue}

Sleepiness occurs early in therapy in $15 \%-35 \%$ of patients but usually resolves (Faught et al 1996; Privitera et al 1996; Tassinari et al 1996). Cognitive effects can occur in the absence of sedation. Fatigue (asthenia) was noted in $13 \%-16 \%$ of child and adult participants in clinical trials (Waugh and Goa 2003; Neurologics 2006). Fatigue may continue indefinitely in some patients.

\section{Carbonic anhydrase inhibition effects}

TPM is a weaker carbonic anhydrase inhibitor than acetazolamide, but may still produce paresthesias, renal stones, and reductions in serum bicarbonate. These effects are related to inhibition of carbonic anhydrase isozymes CA II and CA IV (Dodgson et al 2000). The most common of these is paresthesias. Paresthesias, typically nonpainful tingling in the extremities, occur often in monotherapy; for example in $35 \%-49 \%$ of patients in migraine trials (Neurologics 2006). They are usually transient, do not require drug discontinuation, and patients can be reassured that they are harmless. 


\section{Renal stones}

Renal stones occur in 1\%-2\% of patients (Neurologics 2006). TPM reduces urinary citrate excretion and thus raises urine pH (Sachdeo, Wasserstein et al 2002). There is no clear relationship to dose or to duration of therapy. Previous renal stones are a relative contraindication to TPM. Increased fluid intake may be protective. Routine urinalysis is not recommended; symptoms are usually unmistakable.

\section{Metabolic acidemia}

Metabolic acidemia is a consequence of lowered serum bicarbonate levels, a dose-dependent effect of TPM. An average decrease of $5.1 \mathrm{meq} / \mathrm{L}$ has been reported in adults (Garris and Oles 2005), although the manufacturer states that $32 \%$ of adults taking $400 \mathrm{mg} /$ day had levels of $20 \mathrm{meq} / \mathrm{L}$ or lower (Neurologics 2006). The practical consequences of this are uncertain. It is asymptomatic, but could be significant in the setting of metabolic acidosis from other causes such as diabetes, sepsis, status epilepticus, or the ketogenic diet. Chronic acidemia theoretically could have deleterious long-term effects on bone nutriture, though the effect of TPM on bone health, if any, remains unknown.

\section{Disorders of mood}

Depression is common in epilepsy and affected the same percentage of patients taking either TPM or placebo in the first five clinical trials, about 15\% (Shorvon 1996). Depression, irritability, or aggressiveness affected $12 \%$ of TPM patients in one open-label series (Bootsma et al 2004), and 5\%-6\% in another (Kanner et al 2003). Psychiatric side effects were more common in patients with previous psychiatric histories (Kanner et al 2003).

\section{Psychosis}

There are occasional reports of psychosis apparently precipitated by antiepileptic drugs, and TPM is no exception. Paranoia was the major feature in most of the patients in our experience; psychotic symptoms resolved quickly with TPM discontinuation or dose reduction (Khan et al 1999).

\section{Rash}

TPM has a low rash rate in comparison to other antiepilepsy drugs. About $4 \%$ of patients taking TPM in clinical trials developed rashes, not statistically more than placebo (Neurologics 2006). This is lower than the $8 \%-14 \%$ rate seen with phenytoin, carbamazepine, or lamotrigine (Faught 1999) and it is therefore a good choice for patients who have had rashes on other antiepilepsy agents. There is no discernible risk of life-threatening skin reactions.

\section{Uncommon systemic effects}

Acute secondary angle-closure glaucoma

Acute secondary angle-closure glaucoma occurs rarely (Fraunfelder et al 2004). Routine surveillance is unnecessary because the symptoms-severe pain, redness, and blurred vision - are unlikely to be ignored. This problem begins within a few days of drug initiation, at low doses. It resolves quickly with TPM discontinuation and does not require surgery.

\section{Hypohidrosis}

Hypohidrosis (inappropriately decreased sweating) has been reported in a small number of children and could cause dangerous hyperthermia (Ben-Zeev et al 2003). This may be related to the carbonic anhydrase inhibition (Cerminara et al 2006). Parents should be cautioned about this possibility, especially in hot climates.

\section{Encephalopathy}

Encephalopathy with hyperammonemia has been described in a small number of cases in which TPM was used with concomitant valproate (Hamer et al 2000; Longin et al 2002; Latour et al 2004; Cheung et al 2005). Of, course valproate alone can also produce this effect. Nevertheless, TPM plus valproate is often a useful combination, especially for patients who have gained weight on valproate.

\section{Overdose}

TPM has a wide therapeutic margin and serious effects of overdose occur rarely (Lofton and Klein-Schwartz 2005). Hemodialysis should remove TPM effectively, if necessary, since protein binding is low (Schneiderman 1998).

\section{Use in pregnancy}

The safety of TPM for use during pregnancy has not been established. So far, there are insufficient numbers of women taking TPM enrolled in the various prospective pregnancy registries of antiepilepsy drugs to draw any conclusions. Limb and craniofacial anomalies were observed in the offspring of rodents and rabbits given high doses during gestation (Neurologics 2006). These effects are similar to those seen in animals given acetazolamide, although acetozalamide is not known to be a human teratogen (Nakatsuka et al 1992). This uncertainty should be weighed against the known risk of older drugs and the definite risk, to mother and fetus, of convulsive seizures during pregnancy.

\section{Patient education}

It is not practicable to recite every possible side effect to patients before prescribing, but the possibility of somnolence, 
cognitive or speech slowing, and paresthesias should be mentioned with the qualification that they are usually transient. The $1.5 \%$ kidney stone rate should be stated along with advice to maintain good hydration, especially in hot weather. Advise patients to contact the physician at once with symptoms of glaucoma (eye pain, sudden myopia) or with a rash. Parents of small children should be advised to watch for symptoms of hyperthermia.

\section{Monitoring for side effects}

No routine monitoring of hepatic function or hematologic parameters is required. A baseline serum bicarbonate and repeat measurement at the target dose and after any large dose increases may be considered, though the need for correction is unclear. Both the patient and the family should be questioned about the presence of cognitive or psychiatric adverse effects. There is no battery of specific tests for topiramate toxicity but special attention should be paid to assessment of verbal fluency and expressive speech.

\section{Practical use of topiramate}

TPM is available as 25, 50, 100, and $200 \mathrm{mg}$ tablets and as 15 and $25 \mathrm{mg}$ sprinkle capsules. The capsules can be swallowed or opened and sprinkled on food; the contents may adhere to the walls of tubing so crushed tablets are preferable for administration via feeding tubes. No parenteral form is available.

\section{Initial dosing}

Tolerability is improved by low initial doses with slow titration to the desired target dose in both adults (Biton et al 2001; Dodson et al 2003) and children (Albsoul-Younes et al 2004). Early side effects usually subside within a few weeks (Majkowski et al 2005). Reduction of the dosage of concomitant antiepilepsy drugs is often effective in reducing central nervous system symptoms (Naritoku et al 2005); in one open-label study discontinuations dropped from $23 \%$ to $14 \%$ when other drug dosages were lowered (Dodson et al 2003). Although the manufacturer recommends a starting dose of 50 $\mathrm{mg}$ /day for adults, it is often a better strategy to begin with $25 \mathrm{mg}$ /day with increases no faster than $25 \mathrm{mg} /$ week, unless there is a need for haste. The first-week dose should be given at bedtime, and if the twice-daily dose is asymmetrical, the larger dose should be in the evening. A comparable initial dose for children is 0.5 to $1 \mathrm{mg} / \mathrm{kg} /$ day, with increases of the same amounts at weekly intervals. The monotherapy studies demonstrated the beginning of a significant antiseizure effect even at 50-100 mg/day (Arroyo et al 2005, Gilliam et al 2003), thus patients should have some protection early. On the other hand, larger initial doses do not compromise safety, only tolerability. If the situation warrants, for example with inpatients having active seizures, larger doses such as 100-200 mg/day may be started. This procedure ordinarily should be undertaken in hospital.

\section{Dose targets}

For new-onset epilepsy, a target dose of $100 \mathrm{mg}$ /day has been suggested (Privitera et al 2003). For adjunctive therapy of partial-onset seizures, $200 \mathrm{mg} /$ day was shown to reduce seizure frequency almost as well as higher doses in most patients (Guberman et al 2002). Above $400 \mathrm{mg} /$ day, there was little additional benefit among the population of refractory patients receiving concomitant medications (Faught et al 1996; Privitera et al 1996).

However, individual patients may have better seizure control with doses as high as $1200 \mathrm{mg}$ /day, and increasing the dose gradually to complete control or to clinical toxicity is reasonable if some benefit is noted at lower doses. If no benefit is seen at $400 \mathrm{mg} /$ day, it is unlikely that significant benefit will be seen at higher doses; in that case it makes sense to abandon the drug and try something else even if $400 \mathrm{mg}$ is well-tolerated. In an open-label trial involving 441 patients at 127 sites, primarily in Europe, the average monotherapy dose achieved was $125 \mathrm{mg} /$ day (range 25-700) (Guerrini et al 2005). This trial also enrolled 114 children less than 12 years old; the median final TPM dose reached was $3.3 \mathrm{mg} / \mathrm{kg} /$ day (range 1.3-13.0). Another large naturalistic trial in the United States assessed adjunctive therapy in adults, mostly with enzyme inducing drugs: final doses were most commonly in the 300-350 mg/day range (Dodson et al 2003).

Dosing frequency should be twice daily. In monotherapy or in the absence of enzyme-inducing drugs, once-daily dosing may be allowable. In that case, bedtime dosing is most likely to ameliorate side effects.

Dosing, as with all antiepilepsy drugs, is best adjusted by clinical response rather than by serum level measurements. However, levels are available and can be helpful in some circumstances, for example to assess the effect of enzyme-inducing drugs on topiramate pharmacokinetics or to investigate compliance. Serum levels of 2.5-10.5 micrograms/milliliter are associated with the antiseizure effect (Christensen et al 2003), though some patients will benefit from higher levels and some will do well with lower levels. A withdrawal syndrome has not been described, though it is always prudent to withdraw antiepilepsy drugs gradually. 
Table I

\begin{tabular}{ll}
\hline Advantages & Disadvantages \\
\hline Effective & Cognitive side effects \\
Broad-spectrum & Carbonic anhydrase inhibitor \\
Multiple mechanisms of action & Renal stones \\
Linear pharmacokinetics & Metabolism is inducible \\
Primarily renal excretion & Unknown teratogenicity \\
Not an hepatic enzyme inducer & Slow titration to reduce toxicity \\
Good safety record & Rare serious adverse effects \\
& Expense \\
\hline
\end{tabular}

TPM is relatively expensive in comparison to older drugs, and cost is a barrier to its use in many countries. Pharmacoeconomic analyses are locale-specific and not very useful worldwide. Eventually, generic preparations may result in wider availability.

\section{Summary}

Advantages and disadvantages of topiramate are summarized in Table 1. TPM is a potent drug effective against most seizure types, is remarkably safe, requires little monitoring, and displays a relatively favorable pharmacokinetic profile. It is appropriate for initial monotherapy as well as for monotherapy or adjunctive therapy in refractory patients. It is especially attractive for patients who may also benefit from its anorectic and antimigraine effects. The major problem limiting its use is the frequent occurrence of cognitive adverse effects, especially expressive language dysfunction.

\section{Disclosures}

Dr. Faught has received research support and honoraria for consulting from Johnson and Johnson/OrthoMcNeill, the manufacturer of topiramate, and has received research support or honoraria for speaking and consulting from the manufacturers of the following drugs mentioned in this article: carbamazepine, lamotrigine, oxcarbazepine, phenytoin, and valproate.

\section{References}

Abou-Khalil B. 2000. Topiramate in the long-term management of refractory epilepsy. Topiramate YOL Study Group. Epilepsia, 41 Suppl 1:S72-6.

Albsoul-Younes AM, Salem HA, Ajlouni SF, et al. 2004. Topiramate slow dose titration: improved efficacy and tolerability. Pediatr Neurol, 31:349-52.

Aldenkamp AP. 2000. Cognitive effects of topiramate, gabapentin, and lamotrigine in healthy young adults. Neurology, 54:271-2.

Aldenkamp AP, Baker GA, Mulder OG, et al. 2000. A multicenter, randomized clinical study to evaluate the effect on cognitive function of topiramate compared with valproate as add-on therapy to carbamazepine in patients with partial-onset seizures. Epilepsia, 41:1167-8.

Arroyo S, Dodson WE, Privitera MD, et al. 2005. Randomized dosecontrolled study of topiramate as first-line therapy in epilepsy. Acta Neurol Scand, 112:214-22.
Aykutlu E, Baykan B, Gurses C, et al. 2005. Add-on therapy with topiramate in progressive myoclonic epilepsy. Epilepsy Behav, 6:260-3.

Ben-Menachem E, Axelsen M, Johanson EH, et al. 2003. Predictors of weight loss in adults with topiramate-treated epilepsy. Obes Res, 11:556-62.

Ben-Menachem E, Henriksen O, Dam M, et al. 1996. Double-blind, placebo-controlled trial of topiramate as add-on therapy in patients with refractory partial seizures. Epilepsia, 37:539-43.

Ben-Zeev B, Watemberg N, Augarten A, et al. 2003. Oligohydrosis and hyperthermia: pilot study of a novel topiramate adverse effect. $J$ Child Neurol, 18:254-7.

Bialer M, Doose DR, Murthy B, et al. 2004. Pharmacokinetic interactions of topiramate. Clin Pharmacokinet, 43:763-80.

Biton V, Bourgeois BF. 2005. Topiramate in patients with juvenile myoclonic epilepsy. Arch Neurol, 62:1705-8.

Biton V, Edwards KR, Montouris GD, et al. 2001. Topiramate titration and tolerability. Ann Pharmacother, 35:173-9.

Biton V, Montouris GD, Ritter F, et al. 1999. A randomized, placebocontrolled study of topiramate in primary generalized tonic-clonic seizures. Topiramate YTC Study Group. Neurology, 52:1330-7.

Bootsma HP, Coolen F, Aldenkamp AP, et al. 2004. Topiramate in clinical practice: long-term experience in patients with refractory epilepsy referred to a tertiary epilepsy center. Epilepsy Behav, 5:380-7.

Cerminara C, Seri S, Bombardieri R, et al. 2006. Hypohidrosis during topiramate treatment: a rare and reversible side effect. Pediatr Neurol, $34: 392-4$

Cheung E, Wong V, Fung CW. 2005. Topiramate-valproate-induced hyperammonemic encephalopathy syndrome: case report. $J$ Child Neurol, 20:157-60.

Christensen J, Andreasen F, Poulsen JH, et al. 2003. Randomized, concentration-controlled trial of topiramate in refractory focal epilepsy. Neurology, 61:1210-18.

Coppola G, Capovilla G, Montagnini A, et al. 2002. Topiramate as add-on drug in severe myoclonic epilepsy in infancy: an Italian multicenter open trial. Epilepsy Research, 49:45-8.

Coulter DA, Sombati S, Delorenzo RJ. 1993. Selective effects of topiramate on sustained repetitive firing and spontaneous bursting in cultured hippocampal neurons. Epilepsia, 34:123.

Cross JH. 2002. Topiramate monotherapy for childhood absence seizures: an open-lable pilot study. Seizure, 11:406-10.

Dodgson SJ, Shank RP, Maryanoff BE. 2000. Topiramate as an inhibitor of carbonic anhydrase isoenzymes. Epilepsia, 41 Suppl 1:S35-9.

Dodson WE, Kamin M, Kraut L, et al. 2003. Topiramate titration to response: analysis of individualized therapy study (TRAITS). Epilepsia, $37: 615-20$.

Doose DR, Brodie MJ, Wilson EA, et al. 2003. Topiramate and lamotrigine pharmacokinetics during repetitive monotherapy and combination therapy in epilepsy patients. Epilepsia, 44:917-22.

Doose DR, Walker SA, Gisclon LG, et al. 1996. Single-dose pharmacokinetics and effect of food on the bioavailability of topiramate, a novel antiepileptic drug. J Clin Pharmacol, 36:884-91.

Doose DR, Wang SS, Padmanabhan M, et al. 2003. Effect of topiramate or carbamazepine on the pharmacokinetics of an oral contraceptive containing norethindrone and ethinyl estradiol in healthy obese and nonobese female subjects. Epilepsia, 44:540-9.

Elterman RD, Glauser TA, Wyllie E, et al. 1999. A double-blind, randomized trial of topiramate as adjunctive therapy for partial-onset seizures in children. Topiramate YP Study Group. Neurology, 52:1338-44.

Faught E. 1999. Clinical studies of topiramate. Drugs Today (Barc), $35: 49-57$

Faught E, Limdi N. 2002. Adverse and beneficial side effects of antiepileptic drugs. In Ettinger AB, Devinsky O (eds). Managing epilepsy and co-existing disorders. Boston, Butterworth-Heinemann

Faught E, Wilder BJ, Ramsay RE, et al. 1996. Topiramate placebo-controlled dose-ranging trial in refractory partial epilepsy using 200-, 400-, and $600-\mathrm{mg}$ daily dosages. Topiramate YD Study Group. Neurology, 46:1684-90. 
Fraunfelder FW, Fraunfelder FT, Keates EU. 2004. Topiramate-associated acute, bilateral, secondary angle-closure glaucoma. Ophthalmology, 111:109-11.

Garris SS, Oles KS. 2005. Impact of topiramate on serum bicarbonate concentrations in adults. Annals Pharmacother, 39:424-6.

Gilliam F, Veloso F, Bomhof MA, et al. 2003. A dose-comparison trial of topiramate as monotherapy in recently diagnosed partial epilepsy. Neurology, 60:196-202.

Gisclon LG, Riffits JM, Sica DA, et al. 1993. The pharmacokinetics (PK) of topiramate $(\mathrm{T})$ in subjects with renal impairment (RI) as compared to matched subjects with normal renal function (NRF). Pharmaceutical Research, 10:S397.

Glauser TA. 1999. Topiramate. Epilepsia40 Suppl 5:S71-80.

Glauser TA, Clark PO, Strawsburg R. 1998. A pilot study of topiramate in the treatment of infantile spasms. Epilepsia, 39:1324-8.

Grosso S, Galimberti D, Farnetani MA, et al. 2005. Efficacy and safety of topiramate in infants according to epilepsy syndromes. Seizure, 14:183-9.

Gryder DS, Rogawski MA. 2003. Selective antagonism of GluR5 kainatereceptor-mediated synaptic currents by topiramate in rat basolateral amygdala neurons. J Neuroscience, 23:7069-74.

Guberman A, Neto W, Gassman-Mayer C, et al. 2002. Low-dose topiramate in adults with treatment-resistant partial-onset seizures. Acta Neurol Scand, 106:183-9.

Guerrini R, Carpay J, Groselj J, et al. 2005. Topiramate monotherapy as broad-spectrum antiepileptic drug in a naturalistic clinical setting. Seizure, 14:371-80.

Hamer HM, Knake S, Schomburg U, et al. 2000. Valproate-induced hyperammonemic encephalopathy in the presence of topiramate. Neurology, $54: 230-2$.

Herrero AI, Del Olmo N, Gonzalez-Escalada JR, et al. 2002. Two new actions of topiramate: inhibition of depolarizing GABA(A)-mediated responses and activation of a potassium conductance. Neuropharmacology, 42:210-20.

Hosain SA, Merchant S, Solomon GE, et al. 2006. Topiramate for treatment of infantile spasms. J Child Neurol, 21:17-19.

Jansen J, Aldenkamp AP, Majoie M, et al. 2006. Functional MEI reveals declined prefrontal cortex activation in patients with epilepsy on topiramate therapy. Epilepsy Behav, 9:181-5.

Johannessen SI. 1997. Pharmacokinetics and interaction profile of topiramate: review and comparison with other newer antiepileptic drugs. Epilepsia, 38 Suppl 1:S18-23.

Kanner A, Wuu J, Faught E, et al. 2003. A past psychiatric history may be a risk factor for topiramate-related psychiatric and cognitive adverse events. Epilepsy Behav, 4:548-52.

Khan A, Faught E, Gilliam F, et al. 1999. Acute psychotic symptoms induced by topiramate. Seizure, 8:235-7.

Koh S, Jensen FE. 2001. Topiramate blocks perinatal hypoxia-induced seizures in rat pups. Ann Neurol, 50:366-72.

Korean Topiramate Study Group. 1999. Topiramate in medically intractable partial epilepsies: double-blind placebo-controlled randomized parallel group trial. Epilepsia, 40:1767-74.

Kwan P, Brodie M. 2000. Early identification of refractory epilepsy. N Engl $J$ Med, 342:314-19.

Latour P, Biraben A, Polard E, et al. 2004. Drug induced encephalopathy in six epileptic patients: topiramate? valproate? or both. Hum Psychopharmacol, 19:193-203.

Lee H-W, Jung D-K, Suh C-K, et al. 2006. Cognitive effects of low-dose topiramate monotherapy in epilepsy patients: a 1-year follow-up. Epilepsy Behav, 8:736-41.

Lhatoo SD, Wong IC, Polizzi G, et al. 2000. Long-term retention rates of lamotrigine, gabapentin, and topiramate in chronic epilepsy. Epilepsia, 41:1592-6.

Loescher W, Schmidt D. 2006. Experimental and clinical evidence for loss of effect (tolerance) during prolonged treatment with antiepileptic drugs. Epilepsia, 47:1253-84.

Lofton AL, Klein-Schwartz W. 2005. Evaluation of toxicity of topiramate exposures reported to poison centers. Hum Exp Toxicol, 24:591-5.
Longin E, Teich M, Koelfen W, et al. 2002. Topiramate enhances the risk of valproate-associated side effects in three children. Epilepsia, 43:451-4.

Majkowski J, Neto W, Wapenaar R, et al. 2005. Time course of adverse events in patients with localization-related epilepsy receiving topiramate added to carbamazepine. Epilepsia, 46:648-53.

Marson AG, Al-Kharusi AM, Alwaidh M, et al. 2007a. The SANAD study of effectiveness of carbamazepine, gabapentin, lamotrigine, oxcarbazepine, or topiramate for treamtent of partial epilepsy: an unblinded randomised controlled trial. Lancet, 369:1000-15.

Marson AG, Al-Kharusi AM, Alwaidh M, et al. 2007b. The SANAD study of effectiveness of valproate, lamotrigine, or topiramate for generalised and unclassifiable epilepsy: an unblinded randomised controlled trial. Lancet, 369:1016-26.

Maryanoff BE, Nortey SO, Gardocki JF, et al. 1987. Anticonvulsant O-alkyl sulfamates. 2, 3:4, 5-Bis-O-(1-methylethylidene)-beta-D-fructopyranose sulfamate and related compounds. $J$ Med Chem, 30:880-7.

Mclean MJ, Bukhari AA, Wamil AW. 2000. Effects of topiramate on sodiumdependent action-potential firing by mouse spinal cord neurons in cell culture. Epilepsia, 41 Suppl 1:S21-4.

Meador KJ, Loring DW, Hulihan JF, et al. 2003. Differential cognitive and behavioral effects of topiramate and valproate. Neurology, 60:1483-8.

Meador KJ, Loring DW, Vahle VJ, et al. 2005. Cognitive and behavioral effects of lamotrigine and topiramate in healthy volunteers. Neurology, 64:2108-14.

Mikaeloff Y, De Saint-Martin A, Mancini J, et al. 2003. Topiramate: efficacy and tolerability in children according to epilepsy syndromes. Epilepsy Res, 53:225-32.

Mula M, Trimble MR, Thompson P, et al. 2003. Topiramate and wordfinding difficulties in patients with epilepsy. Neurology, 60:1104-7.

Nakatsuka T, Komatsu T, Fujii T. 1992. Axial skeletal malformations induced by acetazolamide in rabbits. Teratology, 45:629-36.

Naritoku DK, Hulihan JF, Schwarzman LK, et al. 2005. Effect of cotherapy reduction on tolerability of epilepsy add-on therapy: a randomized controlled trial. Ann Pharmacother, 39:418-23.

Neurologics, Ortho-Mcneil Pharmaceutical Inc. 2006. TOPAMAX prescribing information. URL: http://www.topamax-epilepsy.com/utilities/ images/tpamax.pdf\#zoom $=100$

Niebauer M, Gruenthal M. 1999. Topiramate reduces neuronal injury after experimental status epilepticus. Brain Res, 837:263-9.

Nieto-Barrera M, Candau R, Nieto-Jimenez M, et al. 2000. Topiramate in the treatment of severe myoclonic epilepsy in infancy. Seizure, 9:590-4.

Perucca E. 1997. A pharmacological and clinical review on topiramate, a new antiepileptic drug. Pharmacol Res, 35:241-56.

Privitera M, Fincham R, Penry J, et al. 1996. Topiramate placebo-controlled dose-ranging trial in refractory partial epilepsy using 600-, 800-, and 1,000-mg daily dosages. Topiramate YE Study Group. Neurology, 46:1678-83

Privitera MD, Brodie MJ, Mattson RH, et al. 2003. Topiramate, carbamazepine and valproate monotherapy: double-blind comparison in newly diagnosed epilepsy. Acta Neurol Scand, 107:165-75.

Reife R, Pledger G, Wu SC. 2000. Topiramate as add-on therapy: pooled analysis of randomized controlled trials in adults. Epilepsia, 41 Suppl 1:S66-71.

Rigoulot M, Boehrer A, Nehlig A. 2003. Effects of topiramate in two models of genetically-determined generalized epilepsy, the GAERS and the audiogenic Wister AS. Epilepsia, 44:14-19.

Rosenfeld WE, Doose DR, Walker SA, et al. 1999. A study of topiramate pharmacokinetics and tolerability in children withh epilepsy. Pediatric Neurology, 20:339-44.

Rosenfeld WE, Doose DR, Walker SA, et al. 1997. Effect of topiramate on the pharmacokinetics of an oral contraceptive containing norethindrone and ethinyl estradiol in patients with epilepsy. Epilepsia, $38: 317-23$

Rosenfeld WE, Slater J. 2002. Characterization of topiramate-associated weight changes in adults with epilepsy. Epilepsia, 43 (Suppl 7):220-1. 
Sachdeo R, Wasserstein A, Mesenbrink P, et al. 2002. Effects of oxcarbazepine on sodium concentration and water handling. Ann Neurol, 51:613-20.

Sachdeo RC, Glauser TA, Ritter F, et al. 1999. A double-blind, randomized trial of topiramate in Lennox-Gastaut syndrome. Topiramate YL Study Group. Neurology, 52:1882-7.

Sachdeo RC, Reife RA, Lim P, et al. 1997. Topiramate monotherapy for partial onset seizures. Epilepsia, 38:294-300.

Sachdeo RC, Sachdeo SK, Levy RH, et al. 2002. Topiramate and phenytoin pharmacokinetics during repetitive monotherapy and combination therapy to epileptic patients. Epilepsia, 43:691-6.

Sachdeo RC, Sachdeo SK, Walker SA, et al. 1996. Steady-state pharmacokinetics of topiramate and carbamazepine in patients with epilepsy during monotherapy and concomitant therapy. Epilepsia, 37:774-80.

Schneiderman JH. 1998. Topiramate: pharmacokinetics and pharmacodynamics. Can J Neurol Sci, 25:S3-5.

Shank RP, Gardocki JF, Streeter AJ, et al. 2000. An overview of the preclinical aspects of topiramate: pharmacology, pharmacokinetics, and mechanism of action. Epilepsia, 41 Suppl 1:S3-9.

Shank RP, Gardocki JF, Vaught JL, et al. 1994. Topiramate: preclinical evaluation of structurally novel anticonvulsant. Epilepsia, 35:450-60.

Sharief M, Viteri C, Ben-Menachem E, et al. 1996. Double-blind, placebocontrolled study of topiramate in patients with refractory partial epilepsy. Epilepsy Res, 25:217-24.

Shorvon S. 1996. Safety of topiramate: adverse events and relationship to dosing. Epilepsia, 37:S18-22.

Sousa Pda S, De Araujo Filho GM, Garzon E, et al. 2005. Topiramate for the treatment of juvenile myoclonic epilepsy. Arq Neuropsiquiatr, 63:733-7.

Tassinari CA, Michelucci R, Chauvel P, et al. 1996. Double-blind, placebocontrolled trial of topiramate $(600 \mathrm{mg}$ daily) for the treatment of refractory partial epilepsy. Epilepsia, 37:763-8.
Tatum WOT, French JA, Faught E, et al. 2001. Postmarketing experience with topiramate and cognition. Epilepsia, 42:1134-40.

Thijs J, Verhelst H, Van Coster R. 2001. Retrospective study of topiramate in a paediatric population with intractable epilepsy showing promising effects in the West Syndrome patients. Acta Neurol Belg, 101:171-6.

Towne AR, Garnett LK, Waterhouse EJ, et al. 2003. The use of topiramate in refractory status epilepticus. Neurology, 60:332-4.

Valencia I, Fons C, Kothare SV, et al. 2005. Efficacy and tolerability of topiramate in children younger than 2 years old. $J$ Child Neurol, 20:667-9.

Waugh J, Goa KL. 2003. Topiramate: as monotherapy in newly diagnosed epilepsy. CNS Drugs, 17:985-92.

Wauquier A, Zhou S. 1996. Topiramate: a potent anticonvulsant in the amygdala-kindled rat. Epilepsy Res, 24:73-7.

White HS, Brown SD, Woodhead JH, et al. 1997. Topiramate enhances GABA-mediated chloride flux and GABA-evoked chloride currents in murine brain neurons and increases seizure threshold. Epilepsy Res, 28:167-79.

White HS, Brown SD, Woodhead JH, et al. 2000. Topiramate modulates GABA-evoked currents in murine cortical neurons by a nonbenzodiazepine mechanism. Epilepsia, 41 Suppl 1:S17-20.

Wu WN, Heebner JB, Streeter AJ. 1994. Evaluation of the absorption, excretion, pharmacokinetics and metabolism of the anticonvulsant topiramate in healthy men. Pharmaceutical Research, 11:S336.

Zhang X, Velumian AA, Jones OT, et al. 2000. Modulation of high-voltageactivated calcium channels in dentate granule cells by topiramate. Epilepsia, 41:S52-60. 
